\title{
Rate-controlled intraventricular endoscopic irrigation via bipolar foot pedal activation: technical note
}

\author{
Reid Hoshide, MD, MPH, ${ }^{1,2}$ Robert C. Rennert, MD,,2 Carlos E. Sanchez, MD,,2 \\ Joel R. Martin, MD, ${ }^{1,2}$ Vincent J. Cheung, MD, ${ }^{1,2}$ Gayle Gyles, CST, CSFA, ${ }^{1}$ and \\ Michael L. Levy, MD, PhD',2
}

${ }^{1}$ Rady Children's Hospital of San Diego; and 'Department of Neurosurgery, University of California, San Diego, California

\begin{abstract}
Irrigation during intraventricular endoscopic surgery is critical for visualization, with normal intracranial pressure maintained by balancing fluid ingress and egress. Although irrigation is typically achieved through manual manipulation of inexact stopcocks, the authors have developed a rate-controlled, foot pedal-activated system for precise intraventricular irrigation by using a standard irrigating bipolar electrocautery machine.

This study is a retrospective review of patients who underwent endoscopic intraventricular surgery between January 1 , 2018, and September 25, 2019, in which this irrigation system was used. Important components of this system include a bipolar module irrigation regulator that is set to a desired rate, a secure connection of the bipolar irrigation tubing to the endoscope, and one or more open egress ports on the endoscope for passive fluid drainage. Nineteen consecutive patients were identified on review (average age \pm SD, $4.3 \pm 4.1$ years). Procedures performed included third ventriculostomies $(n=10)$; arachnoid/choroid cyst fenestrations/resections $(n=3)$; biopsy/tumor resection $(n=1)$; and combined procedures $(n=5)$. Foot pedal-controlled irrigation provided visualization of all intraventricular structures. A single operator was able to control the endoscope, endoscopic instruments, and irrigation, with assistance as indicated for more complex maneuvers. There were no perioperative complications. Because this setup is easily constructed from a standard irrigating bipolar machine, delivers precise irrigation flow rates, and facilitates a single-surgeon bimanual technique, these data support the utility of foot-controlled irrigation for endoscopic intraventricular surgery.
\end{abstract}

https://thejns.org/doi/abs/10.3171/2019.11.PEDS19471

KEYWORDS neuroendoscopy; pediatric neurosurgery; minimally invasive neurosurgery; surgical safety; surgical technique

I NTRAVENTRICULAR endoscopic surgery requires periodic irrigation to clear debris and maintain adequate visualization of critical intraventricular anatomy. Excessive intraventricular irrigation can nonetheless be dangerous, by causing acute iatrogenic intracranial hypertension if fluid output is not balanced. ${ }^{1-4}$ The manipulation of irrigation flow is typically cumbersome and inexact, however, because it is most often achieved through the use of an in-line stopcock or a control clamp. This setup requires manual manipulation, whereby the surgeon has to either repeatedly pause bimanual endoscopic techniques to personally adjust the flow of irrigation or divert their attention to instruct a surgical assistant in making irrigation adjustments. Direct control of irrigation by the surgeon that preserves bimanual endoscopic technique is more ideal.

To mitigate the risks of excessive irrigation during in- traventricular endoscopic surgery, we propose the use of a rate-controlled, foot pedal-activated irrigation system constructed from the irrigation module of a standard bipolar cautery machine. This setup obviates the need for inexact stopcock or control clamp systems, and gives the surgeon the ability to accurately and independently control irrigation while performing bimanual endoscopic techniques. In this technical note we report our use of a foot pedal-activated, objectively measured irrigation system for endoscopic intraventricular surgery.

\section{Methods}

\section{Study Population}

Retrospective chart review was used to identify neurosurgical patients who underwent endoscopic intraventricu- 
lar surgery between January 1, 2018, and September 25, 2019, with the senior author (M.L.L.) at Rady Children's Hospital of San Diego. The foot pedal-activated irrigation system was used for all endoscopic intraventricular cases performed by the senior author during this timeframe. This study was conducted according to institutional guidelines, Health Insurance Portability and Accountability Act regulations, and review board approval.

\section{Endoscopic Intraventricular Surgery}

To minimize cortical manipulation and tract diameter, access to the ventricular system was typically gained by passing the endoscope directly through a ventricular catheter tract made intraoperatively. In addition to the standard setup for endoscopic intraventricular surgery (including one or more open egress ports on the endoscope for passive fluid drainage), bipolar irrigation tubing was hooked up to an irrigating bipolar module (Kirwan Surgical Products, LLC) in the normal fashion. The slip-tip male end of the bipolar irrigation tubing was then fit snugly into one of the female Luer-lock irrigating ports of a pediatric Aesculap endoscope (PaediScope [outer diameter $3.0 \mathrm{~mm}$ ], Aesculap, Inc.) (Fig. 1A). The other drainage port is left open to allow for passive egress of CSF and irrigation fluid, and the center instrument port is used normally. The Kirwan bipolar module used in our facility allows for the selection of irrigation run rates between machine settings of 0 and $50 \mathrm{ml} / \mathrm{min}$ (Fig. 1B), with a baseline rate set at the beginning of the case. Importantly, no cautery forceps were connected to the cautery output of the bipolar machine to prevent injury or fire risk with inadvertent activation of electrocautery while using the bipolar pedal for irrigation.

During initial usage of this system, multiple irrigation settings up to the $50 \mathrm{ml} / \mathrm{min}$ maximum on the Kirwan bipolar irrigation module were tested, with a machine setting of $50 \mathrm{ml} / \mathrm{min}$ found to provide optimal clearance of debris and appropriate visualization of intraventricular structures in most cases. Intraoperative testing of the actual irrigation flow rate nonetheless demonstrated a true delivery rate of $20 \mathrm{ml} / \mathrm{min}$ at this setting. During normal use of the endoscope for intraventricular surgery, $10 \mathrm{ml}$ of CSF was drained after placement of the ventricular catheter prior to the endoscopic portion of the case, providing approximately 30 seconds of subsequent irrigation time before reaching the patient's baseline fluid balance. Throughout the intraventricular portion of the procedure, the primary surgeon depressed the foot pedal to activate irrigation when desired (Fig. 1C). Irrigation was used judiciously overall, with no baseline inflow when the foot pedal was not activated. Calculation of the total volume of irrigation administered was possible intraoperatively based on a real-time display of total "bipolar" activation time on the bipolar module and the empirically determined flow rate. Although not needed in this series after the initial optimization period, modulation of irrigation rates is easily achieved in real time by a circulator changing the settings on the bipolar module.

\section{Statistical Analysis}

The mean \pm SD is reported for continuous variables.

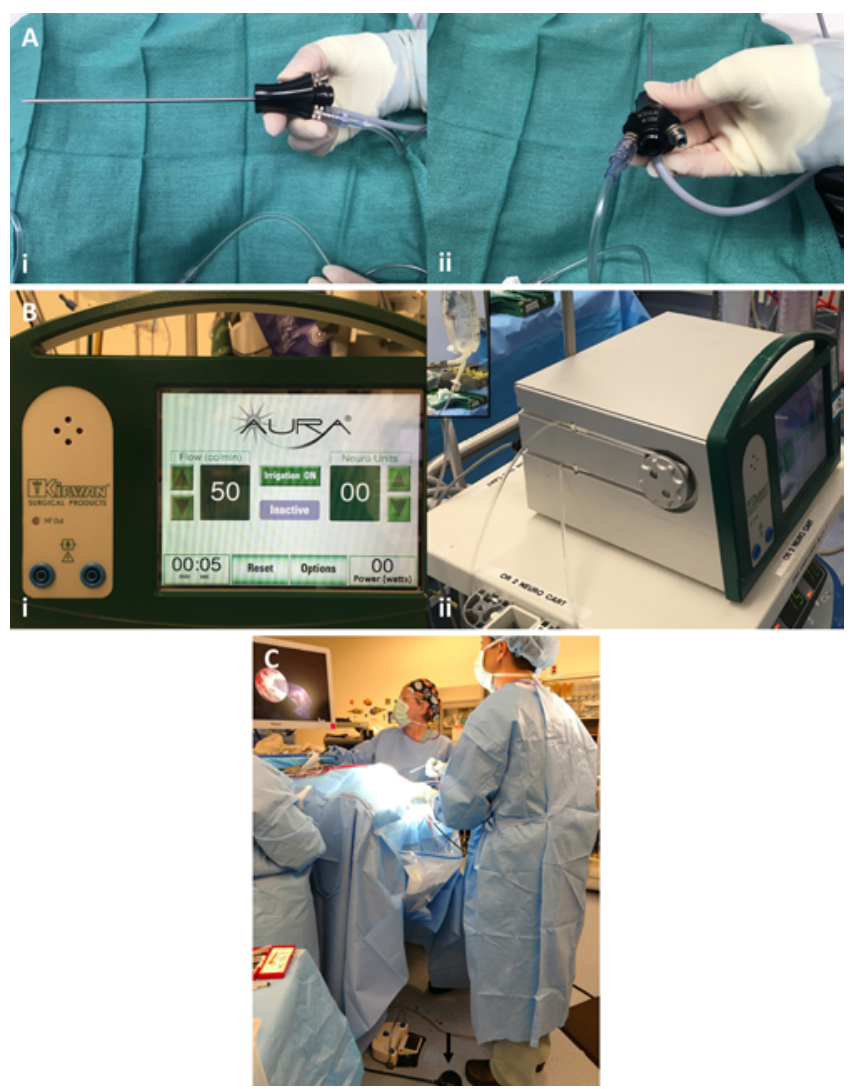

FIG. 1. Setup of rate-controlled, foot pedal-activated irrigation system for endoscopic intraventricular surgery. A: The slip-tip male end of bipolar irrigation tubing is fit snugly within the Luer-lock irrigation port of an Aesculap endoscope (i and ii). The other drainage port is left open to allow for passive egress of CSF and irrigation fluid. The center instrument port is used as needed. B: Off of the surgical field, the other end of the bipolar irrigation tubing is fit into a standard Kirwan bipolar irrigation and electrocautery module (i and ii; inset in ii depicts irrigation bag). No cautery forceps are connected to the cautery output of the bipolar machine to prevent injury or fire risk with bipolar pedal activation for irrigation. The bipolar module has a touch-screen interface that can dial the irrigation rate from 0 to $50 \mathrm{ml} / \mathrm{min}$. In the bottom portion of the interface, the total amount of bipolar activation time is logged, enabling the surgeon to determine the total volume of irrigation used in real time. C: With this setup, a single operator is able to bimanually manipulate the endoscope and the endoscopic instruments, with the irrigation controlled by the foot pedal seen by the operator's left foot (highlighted by the black arrow). Figure is available in color online only.

\section{Results}

Nineteen consecutive patients (11 girls, 8 boys) underwent endoscopic intraventricular surgery using a rate-controlled, foot pedal-activated irrigation system during the study period. The average patient age was $4.3 \pm 4.1$ years. Procedures performed included 10 endoscopic third ventriculostomies (ETVs), 3 endoscopic arachnoid/choroid cyst fenestrations/partial resections, 1 endoscopic biopsy/ tumor resection, and 5 combined procedures (3 ETVs/endoscopic cyst fenestrations, 2 ETVs/endoscopic biopsies with or without tumor debulking) (Table 1). A baseline irrigation machine setting of $50 \mathrm{ml} / \mathrm{min}$ (an empirically determined true irrigation rate of $20 \mathrm{ml} / \mathrm{min}$ ) effectively 
TABLE 1. Summary of characteristics in 19 pediatric patients who underwent endoscopic intraventricular surgery

\begin{tabular}{|c|c|c|}
\hline $\begin{array}{l}\text { Age } \\
\text { (yrs) }\end{array}$ & Sex & Operation \\
\hline 11 & M & $\begin{array}{l}\text { Endoscopic fenestration/partial resection of rt atrial } \\
\text { choroid plexus cyst }\end{array}$ \\
\hline 11 & $\mathrm{~F}$ & Endoscopic biopsy \& resection of rt lateral ventricle tumor \\
\hline 0.9 & $\mathrm{~F}$ & Endoscopic fenestration of It atrial arachnoid cyst \\
\hline 4 & $\mathrm{~F}$ & $\begin{array}{l}\text { Endoscopic fenestration of It fronto-temporal arachnoid } \\
\text { cyst }\end{array}$ \\
\hline 0.5 & M & ETV \\
\hline 0.8 & $\mathrm{~F}$ & ETV \\
\hline 0.9 & M & ETV \\
\hline 1 & M & ETV \\
\hline 1.6 & M & ETV \\
\hline 2 & M & ETV \\
\hline 4 & $\mathrm{~F}$ & ETV \\
\hline 5 & $\mathrm{~F}$ & ETV \\
\hline 6 & M & ETV \\
\hline 10 & $\mathrm{~F}$ & ETV \\
\hline 2 & $\mathrm{~F}$ & $\begin{array}{l}\text { ETV/endoscopic biopsy \& debulking of It exophytic } \\
\text { thalamic tumor }\end{array}$ \\
\hline 12 & $\mathrm{~F}$ & ETV/endoscopic biopsy of third ventricular lesion \\
\hline 0.8 & $\mathrm{~F}$ & $\begin{array}{l}\text { ETV/endoscopic fenestration of posterior fossa arachnoid } \\
\text { cyst }\end{array}$ \\
\hline 8 & M & $\begin{array}{l}\text { ETV/endoscopic fenestration of rt fronto-temporo-parietal } \\
\text { arachnoid cyst }\end{array}$ \\
\hline 1.2 & $\mathrm{~F}$ & $\begin{array}{l}\text { ETV/endoscopic fenestration of suprasellar arachnoid } \\
\text { cyst }\end{array}$ \\
\hline
\end{tabular}

cleared debris and provided adequate visualization of intraventricular structures (Fig. 2). There were no leaks around the junction where the bipolar tubing connected with the Aesculap endoscopic irrigation port. Distal irrigation at the tip of the endoscope was reliable. A single operator was able to control the endoscope and endoscopic instruments bimanually and irrigation with the foot pedal, with additional assistance as indicated for more complex maneuvers (Video 1).

VIDEO 1. Step-by-step use and case examples of the footcontrolled irrigation system for endoscopic intraventricular surgery. 0:10-Irrigation and endoscopy setup. 0:40-Case example 1.

1:58-Case example 2. Copyright Michael L. Levy. Published with permission. Click here to view.

There were no intraoperative signs of increased intracranial pressure (ICP) or perioperative complications.

\section{Illustrative Case}

A 9-month-old otherwise healthy girl with a known posterior fossa arachnoid cyst first seen on prenatal ultrasound was noted to have a rapidly increasing head circumference (from 37th percentile at birth to the 95th percentile), with cyst expansion causing progressive brainstem compression and hydrocephalus on serial imaging (Fig. 3). Despite meeting all developmental milestones and
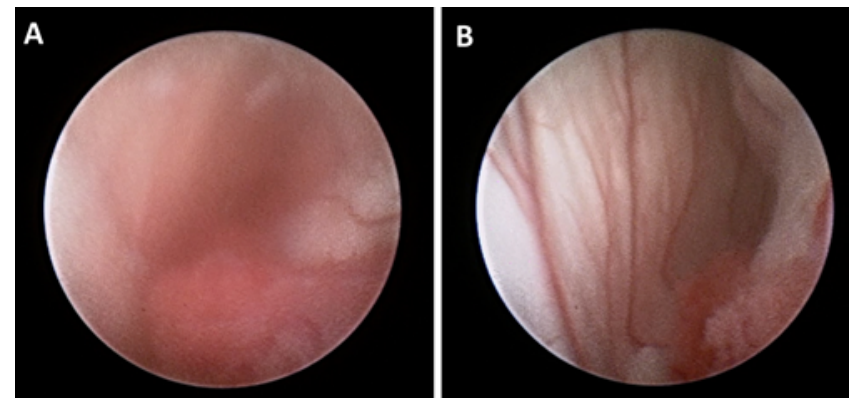

FIG. 2. Controlled intraventricular irrigation for improved endoscopic visualization. At times when hemorrhage or debris obscures the endoscopic field (A), foot pedal-activated irrigation allows the surgeon to rapidly improve visualization with a limited volume of irrigant (B). Figure is available in color online only.

showing no clinical signs of increased ICP, a combined ETV and cyst fenestration was recommended to address the progressive hydrocephalus and compression of local neurological structures.

After intubation, positioning, and registration of neuronavigation, an Aesculap endoscope with 2 Luer-lock ports, a center instrument port, and foot-controlled bipolar irrigation was set up. A right-side burr hole was drilled at a point predetermined by neuronavigation. The occipital horn of the right lateral ventricle was accessed using a ventriculostomy catheter with navigation, $10 \mathrm{ml}$ of CSF was drained, and the catheter was removed. The endoscope was then advanced through this same corridor. Foot pedal-controlled irrigation, set at $50 \mathrm{ml} / \mathrm{min}$ (true irrigation rate of $20 \mathrm{ml} / \mathrm{min}$ ), was activated by the surgeon as needed as the endoscope was advanced through the cerebrum into the lateral ventricle. The other instrument ports were left open to allow for passive egress of CSF and irrigation fluid. Once in the lateral ventricle, anatomical landmarks and neuronavigation were used to identify the superior cyst wall, which was perforated using an alligator clamp, and the fenestration was expanded with a Fogarty balloon. The endoscope was then passed through this hole into the cyst cavity in the posterior fossa, and similar fenestration of the deep cyst wall was performed. At the conclusion of this procedure, CSF was seen flowing through both fenestrations. The endoscope was removed and the wound was closed. A similar technique was then used to perform an ETV with a new right frontal burr hole.

Throughout the case, judicious irrigation was used as needed to clear the field of debris for appropriate visualization of critical structures. There were no physiological signs of acute intracranial hypertension (bradycardia/ tachycardia or hypertension), ${ }^{3}$ hemorrhage, or technical difficulties with the bipolar irrigation module or endoscope. The patient had an uneventful postoperative course. At the 4-month follow-up she continued to meet all developmental milestones with close monitoring of head circumference and serial imaging planned.

\section{Discussion}

A well-known complication of intraventricular endo- 

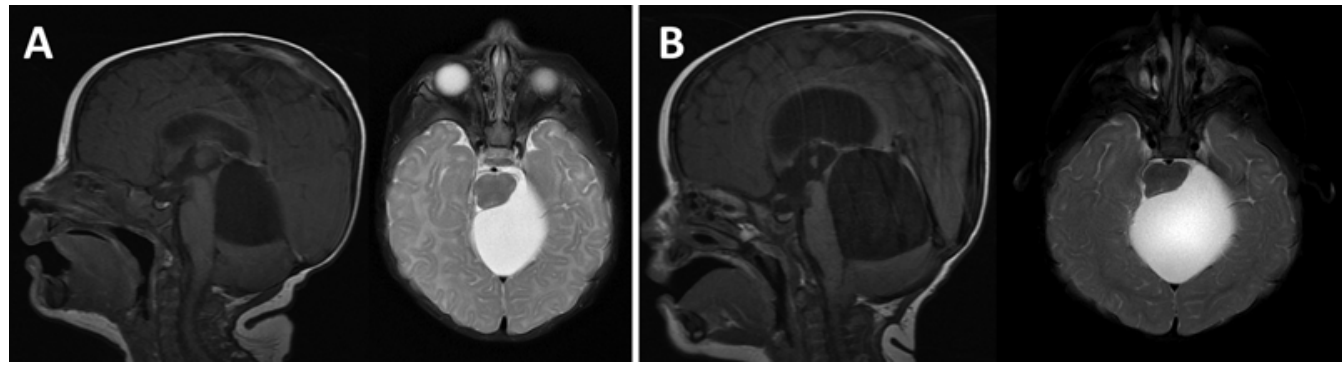

FIG. 3. Illustrative case. A 9-month-old healthy girl with a posterior fossa arachnoid cyst first seen on prenatal ultrasound was initially managed conservatively. A: MRI sequence obtained at 5 months demonstrated a $4.6 \times 4.0 \times 4.2-\mathrm{cm}$ posterior fossa cyst with mild brainstem compression and noncommunicating hydrocephalus (sagittal T1 [left] and axial T2 [right] images). Despite meeting all developmental milestones, she experienced notable head growth. B: MRI sequence obtained at 9 months demonstrated cyst expansion to $5.9 \times 5.0 \times 5.0 \mathrm{~cm}$ with significantly increased brainstem compression and hydrocephalus (sagittal T1 [left] and axial T2 [right] images). She underwent an uneventful combined endoscopic cyst fenestration and ETV performed using the rate-controlled, foot pedal-activated irrigation system. Close clinical follow-up is ongoing.

scopic surgery is acute elevations in ICP from excessive irrigation without adequate drainage. ${ }^{3,4}$ If not anticipated, this imbalance in ingress and egress can be rapidly fatal, ${ }^{1,2}$ especially with the use of inadequately controlled pressurized fluid sources, which are often used to improve visibility and clearance of debris as compared to passive gravity irrigation. Current means of inflow rate control including a stopcock or a control clamp are inexact, and require manual manipulation that can result in long or continuous "on" periods and are subjectively controlled by feel. Fluid egress is typically passive and is maintained by using a peel-away sheath with a larger diameter than the endoscope, or by leaving endoscope instrument ports open. However, with either technique excessive irrigation can overwhelm passive outflow and increase ICP.

The bipolar irrigation system described in this series provides dynamically controlled, precise levels of intraventricular irrigation through foot pedal activation. Representing an evolution from gravity or pressure-bag irrigation delivery approaches previously used at our institution, this setup allows the surgeon to deliver irrigation only when needed to clear debris from the visual field, without diverting attention from the operation to adjust flow rates or compromise the bimanual technique. This technique minimizes total irrigation volume to the intracranial space during endoscopic surgeries. When combined with our practice of draining $10 \mathrm{ml}$ of CSF at the beginning of the case (empirically equivalent to 30 seconds of irrigation time at our preferred irrigation setting), the risk of irrigation-linked intraoperative ICP crises is minimized while maintaining excellent intraventricular visualization. In situations in which continuous irrigation is needed to clear heavy debris or persistent intraventricular hemorrhage, this is easily accomplished by sustained foot pedal activation with continuous open egress of fluid through the endoscope port maintaining physiological ICP (Fig. 2).

Whereas either the use of a peel-away sheath or the maintenance of open endoscope instrument ports allows for passive drainage during intraventricular surgery, we prefer the latter method in an attempt to minimize cortical and white matter trauma. Specifically, passage of the 3.0-mm-diameter pediatric endoscope under direct visual- ization down a tract from a standard round-tip ventricular catheter as described above limits the resulting tract size to the diameter of the endoscope, with less theoretical tissue disruption than a direct pass of the flat-tip endoscope and a smaller footprint than the approximately 3.3- to 4.4$\mathrm{mm}$ or greater cortical tract diameter associated with the use of an operating sheath.

Because this approach adapts a standard irrigating bipolar machine for another surgical use, it is not surprising that there are differences in the set and delivered irrigation flow rates. Empirical testing and optimization of the delivered flow rate by surgeons adapting this approach should thus be performed, especially if using a different bipolar machine, endoscope, and/or an operating sheath for fluid egress. The presence of irrigating bipolar machines in the majority of neurosurgical operating rooms worldwide, however, suggests the potential widespread applicability of this technique.

\section{Conclusions}

Delivery of foot pedal-controlled, metered irrigation during endoscopic intraventricular surgery is easily achieved through adaption of a standard irrigating bipolar machine. This setup promotes judicious use of irrigation to limit the risk of intraoperative intracranial hypertension, while allowing the surgeon to maintain a bimanual endoscopic technique.

\section{References}

1. Fàbregas N, López A, Valero R, Carrero E, Caral L, Ferrer $\mathrm{E}$ : Anesthetic management of surgical neuroendoscopies: usefulness of monitoring the pressure inside the neuroendoscope. J Neurosurg Anesthesiol 12:21-28, 2000

2. Fàbregas N, Valero R, Carrero E, Tercero J, Caral L, Zavala $\mathrm{E}$, et al: Episodic high irrigation pressure during surgical neuroendoscopy may cause intermittent intracranial circulatory insufficiency. J Neurosurg Anesthesiol 13:152-157, 2001

3. Kalmar AF, Van Aken J, Caemaert J, Mortier EP, Struys MM: Value of Cushing reflex as warning sign for brain ischaemia during neuroendoscopy. Br J Anaesth 94:791-799, 2005 
4. van Aken J, Struys M, Verplancke T, de Baerdemaeker L, Caemaert J, Mortier E: Cardiovascular changes during endoscopic third ventriculostomy. Minim Invasive Neurosurg 46:198-201, 2003

\section{Disclosures}

The authors report no conflict of interest concerning the materials or methods used in this study or the findings specified in this paper.

\section{Author Contributions}

Conception and design: Levy, Hoshide, Rennert, Gyles. Acquisition of data: Hoshide, Rennert. Analysis and interpretation of data: Levy, Hoshide, Rennert, Martin. Drafting the article: all authors. Critically revising the article: all authors. Reviewed submitted version of manuscript: Levy, Hoshide, Rennert, Sanchez, Cheung, Gyles. Approved the final version of the manuscript on behalf of all authors: Levy.

\section{Supplemental Information \\ Videos}

Video 1. https://vimeo.com/372365428.

\section{Correspondence}

Michael L. Levy: University of California, San Diego, CA. mlevy@rchsd.org. 